\title{
Obvious Reduction of the Tumor Size in Dedifferentiated Liposarcoma in a Lung Metastasis Patient Receiving Individualized Treatment
}

\author{
Xin-Li Wang Jia-Yao Gong Yan Xue \\ Department of Oncology, Xi'an International Medical Center Hospital, Shanxi, China
}

Keywords

Dedifferentiated liposarcoma $\cdot$ CDK4 amplification · Palbociclib · Eribulin · Lenvatinib

\begin{abstract}
Abdominal metastasis is relatively rare in dedifferentiated liposarcoma of the shoulder and back. Surgery is the best treatment option, whether it is radical or palliative surgery. Chemotherapy is the standard systemic treatment for advanced unresectable/metastatic patients, but the therapeutic effect is limited. Here, we treat advanced abdominal dedifferentiated liposarcoma through a comprehensive treatment method of targeting, surgery, and chemotherapy, which improves the quality of life of the patient, and shrinks the tumor significantly.

(c) 2021 The Author(s).

Published by S. Karger AG, Basel
\end{abstract}

\section{Introduction}

In the WHO classification criteria for bone and soft tissue tumors, dedifferentiated liposarcoma (DDLPS) is defined as a typical lipomatous tumor/well-differentiated liposarcoma (WDLPS) dedifferentiated into sarcomas of different degrees. The differentiated components are mostly high-grade sarcomas, such as pleomorphic undifferentiated sarcoma, fibrosarcoma, and myxofibrosarcoma, etc., and there are also low-grade sarcoma components [1]. DDLPS is a group of rare and highly heterogeneous tumors, which are characterized by local invasiveness, invasive or destructive growth, and a high recurrence rate, and for which radical surgery is the main recommended treatment method [2]. Although surgical resection is currently the main treatment method, the effect is very limited for patients with recurrence 


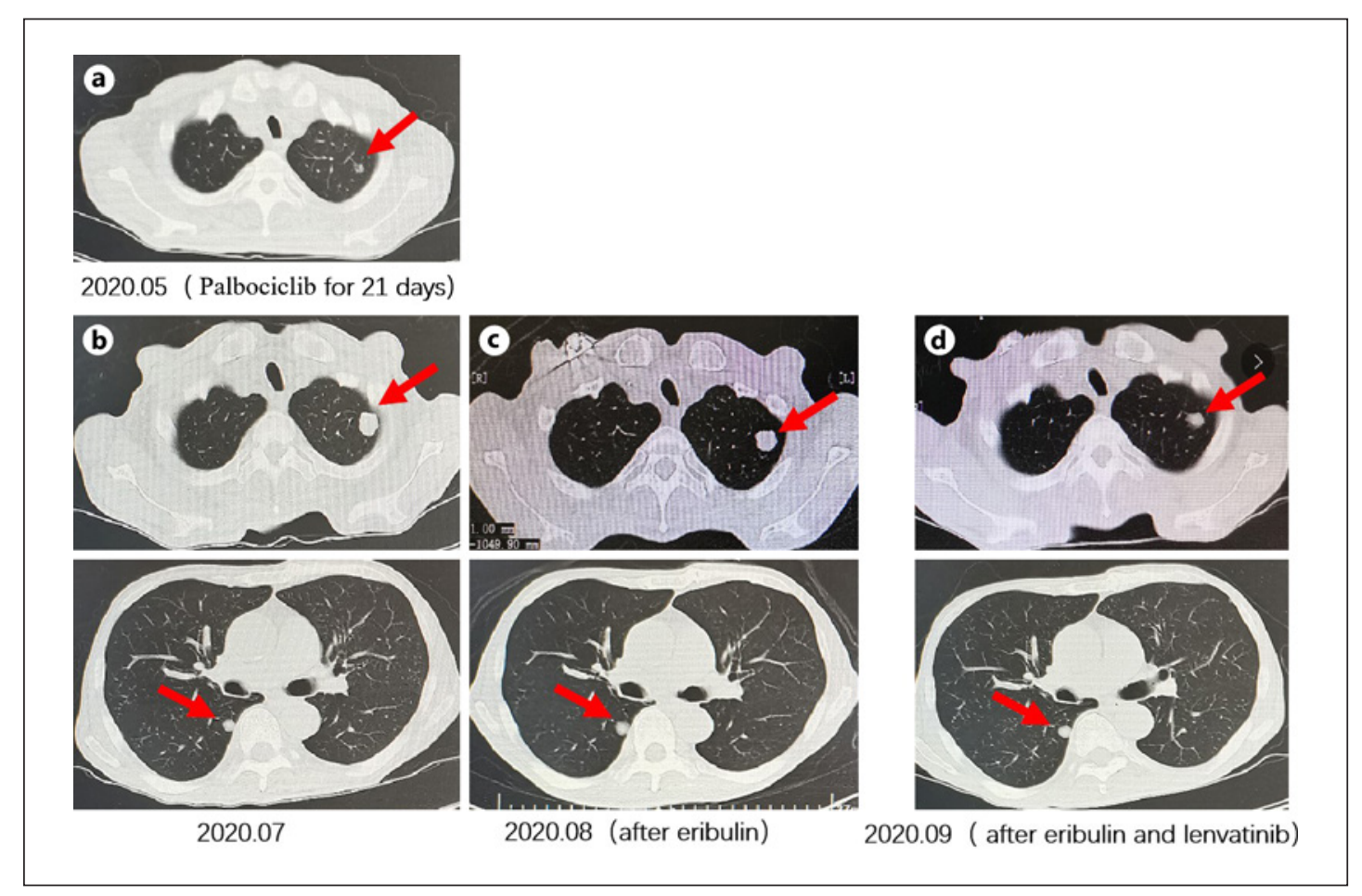

2020.07

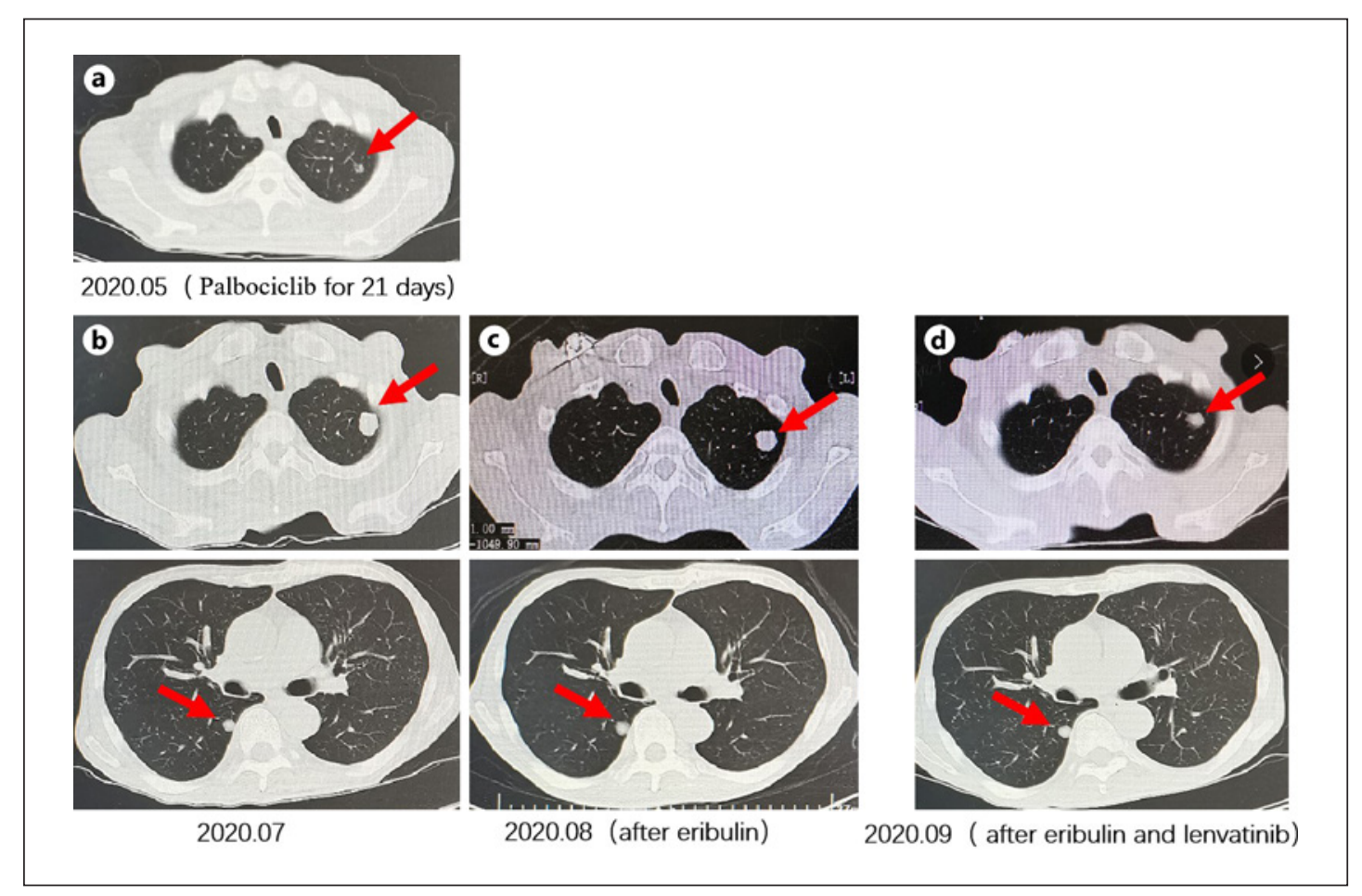

Fig. 1. a CT showed a new left lung nodule after palbociclib treatment, indicating the progress of the disease. b Chest CT showed the metastases of the left upper lobe and the right lower lobe before treatment with eribulin. c After 2 cycles of eribulin treatment, chest CT showed stable lung metastases. d After eribulin combined with lenvatinib, chest CT showed the left lung metastasis to be reduced.

or metastasis of DDLPS. In the past few decades, the prognosis of patients with inoperable, advanced, or metastatic DDLPS has not been significantly improved, and there is an unmet medical need for DDLPS.

\section{Case Presentation}

A 64-year-old male patient underwent a mass resection of the right shoulder and back more than a year ago. The pathology afterward showed DDLPS. The patient refused to undergo a second operation, and did not have regular follow-ups. In November 2019, a PET/CT examination revealed a recurrence of the abdominal cavity. On November 27, 2019, abdominal liposarcoma resection and right hemicolectomy were performed. The postoperative pathology suggested DDLPS of the back mass. The immunohistochemistry was as follows: ALL (-), AE1/ AE3 (-), Bcl-2 (-), CD10 (+), CD34 (-), CD99 (pulp granular), CR (-), desmin $(-)$, EMA $( \pm)$, H-cald (-), HMB-45 (-), Pan-mel (-), S-100 (-), SMA (-), Vim (+), Ki-67 (70\%). The FISH prompt indicated MDM2 gene amplification, and the proportion of positive cells was $60 \%$. The patient did not receive postoperative adjuvant treatment. Five months ago, he was admitted to our hospital with bloody stool. Due to repeated blood in the stool, his general condition was poor. The NGS indicated CDK4/6 amplification.

Combining the patient's general condition and genetic testing result, he was given palbociclib treatment. After 21 days of treatment with palbociclib, a new left lung nodule was found. Due to repeated hematochezia, he underwent resection of a giant abdominal tumor plus 


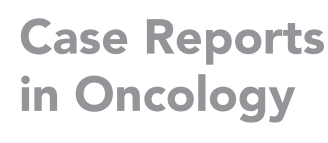

\begin{tabular}{l|l}
\hline Case Rep Oncol 2021;14:1080-1084 \\
\hline DOI: 10.1159/000512519 & $\begin{array}{l}\text { @ 2021 The Author(s). Published by S. Karger AG, Basel } \\
\text { www.karger.com/cro }\end{array}$ \\
\hline
\end{tabular}

Wang et al.: Individualized Treatment for Dedifferentiated Liposarcoma

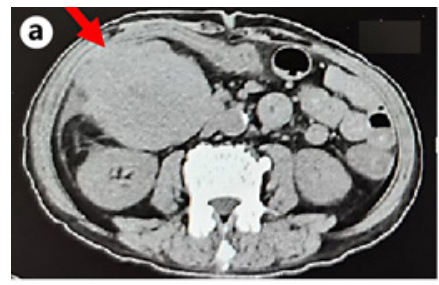

2020.04

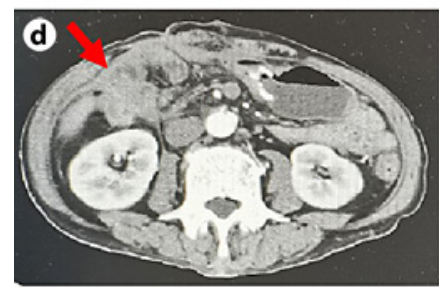

2020.07

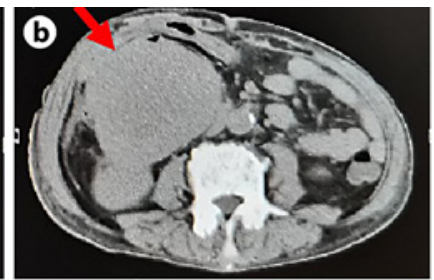

2020.05 (Palbociclib for 21 days)

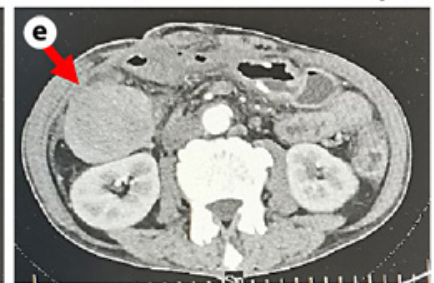

2020.08 (after eribulin)
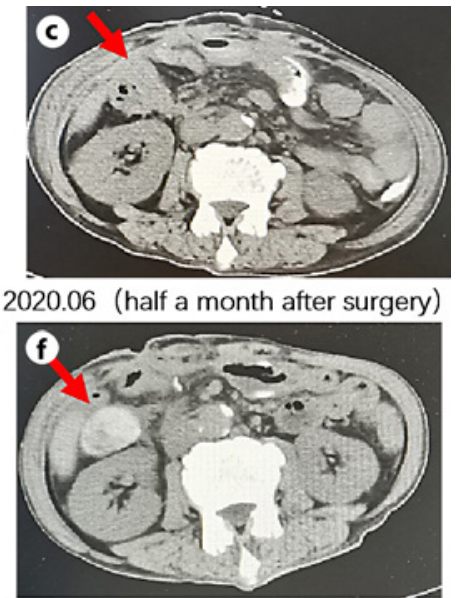

2020.09 ( after eribulin and lenvatinib)

Fig. 2. a Abdominal CT results when the patient was admitted to our hospital for the first time. b Abdominal CT showed that the abdominal lesion was stable after palbociclib treatment for more than 20 days. c, d Abdominal CT half a month after surgery and more than 1 month after surgery showed abdominal recurrence. e The abdominal lesion increased after 2 cycles of eribulin treatment. f Abdominal lesions decreased significantly after eribulin combined with lenvatinib treatment.

bowel resection plus cholecystectomy plus release of intestinal adhesions in our hospital on May 15, 2020. More than 1 month after the operation, the abdominal cavity recurred again, and he was given 2 cycles of "eribulin $2 \mathrm{mg} \mathrm{d} 1,8$ " chemotherapy. After evaluating the progress of the disease, the regimen was changed to "eribulin $2 \mathrm{mg} \mathrm{d1,8"} \mathrm{combined} \mathrm{with} \mathrm{"lenvatinib} 8$ mg" treatment. Recently, it was evaluated that the abdominal mass had significantly retreated and the lung nodules were reduced (stable disease by RECIST 1.1, shown in Fig. 1, 2).

\section{Discussion}

DDLPS accounts for about 18\% of liposarcomas and was described by Evans in 1979. Most DDLPS are primary tumors (80-90\%), and secondary dedifferentiation can occur in $10 \%$ of cases, mostly related to multiple recurrences of WDLPS [3]. DDLPS mostly affects middle-aged and elderly patients, and there is no significant gender difference. The incidence is diverse, with the most common being in the retroperitoneum, but it can also occur in the mediastinum, thoracic cavity, abdominal pelvic cavity, trunk and limbs, groin, and skin, etc. [4]. MDM2 is the most common amplified gene of DDLPS, which is expressed in almost $100 \%$ of WDLPS and DDLPS, and the amplified expression rate of the CDK4 gene exceeds $90 \%$ [3]. The high amplification of MDM2 and CDK4 indicates a poor prognosis [5]. The detection of MDM2 amplification by FISH is the gold standard for diagnosis of WDLPS and DDLPS.

A phase II clinical trial of palbociclib in the treatment of patients with advanced welldifferentiated DDLPS showed that the proportion of patients with progression-free survival (PFS) $>12$ weeks was 66\% [6]. The patient's genetic test revealed CDK4 amplification; 125 $\mathrm{mg}$ of palbociclib q.d. was given for 21 days, with re-examination of stable abdominal masses and new left lung nodules to determine the progress of the disease. Due to repeated blood in the stool, palliative surgery was performed to remove the abdominal mass. Chemotherapy is 
the basis for the treatment of advanced soft tissue sarcoma. At present, adriamycin \pm ifosfamide is the first-line option, and the second-line drugs include gemcitabine, dacarbazine, eribulin, trabectedin, and others. Past studies have shown an overall response rate (ORR) between 3.9 and 20\%, and mean PFS between 3.6 and 6.8 months [7-10]. The 2020 ASCO 11507 LEADER study, a phase Ib/II study of lenvatinib combined with eribulin in the treatment of liposarcoma and leiomyosarcoma, had an ORR 28.5\%, median PFS of 8.6 months, and the combination of lenvatinib and eribulin showed promising effectiveness in the treatment of liposarcoma and leiomyosarcoma [11]. As a new type of microtubule inhibitor, eribulin can induce vascular remodeling, reduce the tumor hypoxic environment, and promote epithelial cell transformation. The 309 study confirmed that eribulin can significantly improve the overall survival of patients with advanced liposarcoma, and further analysis showed that its impact on life is less than that of dacarbazine [9]. Lenvatinib is a multi-target receptor tyrosine kinase inhibitor that can block a series of regulatory factors including VEGFR1-3, FGFR1-4, PDGFR $\alpha, K I T$, and RET in tumor cells. Lenvatinib has a dazzling performance in a variety of cancer types. After single-agent eribulin treatment the abdominal mass progressed; thus, based on the LEADER study, eribulin combined with lenvatinib was given. The patient's mass receded significantly, with controllable third-degree myelosuppression, moderate anemia, and mild fatigue. After multi-line systemic treatment and surgical treatment, the application of eribulin combined with lenvatinib has significantly reduced the abdominal mass and reduced lung metastases, with significant effects. This report offers useful experience for advanced DDLPS.

\section{Conclusion}

Comprehensive, precise, and individualized treatment can effectively treat DDLPS of the abdominal cavity.

\section{Statement of Ethics}

The patient gave his written informed consent to publish his case (including the publication of images).

\section{Conflict of Interest Statement}

The authors have no conflicts of interest to declare.

\section{Funding Sources}

This work was not supported by any foundation.

\section{Author Contributions}

X.-L.W. treated the patient and designed and wrote this research. Y.X. directed the writing of the paper. J.-Y.G. contributed to the collection of images. All authors critically reviewed and approved the final version of the manuscript. 


\section{References}

1 Doyle LA. Sarcoma classification: an update based on the 2013 World Health Organization Classification of Tumors of Soft Tissue and Bone. Cancer. 2014;120(12):1763.

2 Carboni F, Valle M, Federici O, Covello R, Garofalo A. Giant primary retroperitoneal dedifferentiated liposarcoma. J Gastrointest Surg. 2019;23(7):1521-3.

3 Ben Salha I, Zaidi S, Noujaim J, Miah AB, Fisher C, Jones RL, et al. Rare aggressive behavior of MDM2-amplified retroperitoneal dedifferentiated liposarcoma, with brain, lung and subcutaneous metastases. Rare Tumors. 2016;8(3):6282.

4 Byun JY, Choi YW, Choi HY, Myung KB. A case of dedifferentiated liposarcoma that developed in the dermis. Ann Dermatol. 2016;20(4):204-8.

5 Ricciotti RW, Baraff AJ, Jour G, Kyriss M, Wu Y, Liu Y, et al. High amplification levels of MDM2 and CDK4 correlate with poor outcome in patients with dedifferentiated liposarcoma: a cytogenomic microarray analysis of 47 cases. Cancer Genet. 2017;218-219:69-80.

6 Dickson MA, Tap WD, Keohan ML, D'Angelo SP, Gounder MM, Antonescu CR, et al. Phase II trial of the CDK4 inhibitor PD0332991 in patients with advanced CDK4-amplified well-differentiated or dedifferentiated liposarcoma. J Clin Oncol. 2013;31(16):2024-8.

7 Tap WD, Wagner AJ, Schöffski P, Martin-Broto J, Krarup-Hansen A, Ganjoo KN, et al. Effect of doxorubicin plus olaratumab vs doxorubicin plus placebo on survival in patients with advanced soft tissue sarcomas: the ANNOUNCE Randomized Clinical Trial. JAMA. 2020 Apr 7;323(13):1266-76.

8 B Seddon, SJ Strauss, J Whelan, Leahy M, Woll PJ, Cowie F, et al. Gemcitabine and docetaxel versus doxorubicin as first-line treatment in previously untreated advanced unresectable or metastatic soft-tissue sarcomas (GeDDiS): a randomised controlled phase 3 trial. Lancet Oncol. 2017;18(10):1397-410.

9 Schöffski P, Chawla S, Maki RG, Italiano A, Gelderblom H, Choy E, et al. Eribulin versus dacarbazine in previously treated patients with advanced liposarcoma or leiomyosarcoma: a randomised, open-label, multicentre, phase 3 trial. Lancet. 2016;387(10028):1629-37.

10 Demetri GD, von Mehren M, Jones RL, Hensley ML, Schuetze SM, Staddon A, et al. Efficacy and safety of trabectedin or dacarbazine for metastatic liposarcoma or leiomyosarcoma after failure of conventional chemotherapy: results of a phase III randomized multicenter clinical trial. J Clin Oncol. 2016;34(8):786-93.

11 ASCO 11507 LEADER study: Phase Ib/II study of Lenvatinib (L) combined with Eribulin (E) in the treatment of liposarcoma (LPS) and leiomyosarcoma (LMS). 